\title{
Bit-Error-Rate Measurements of RSFQ Shift Register Memories
}

\author{
Tomohiro Hikida, Kan Fujiwara, Nobuyuki Yoshikawa, Akira Fujimaki, Hirotaka Terai, and Shinichi Yorozu
}

\begin{abstract}
Error rates of rapid-single-flux-quantum (RSFQ) shift register memories were investigated using a high-speed error-rate measurement system in order to demonstrate their reliability and stability. We designed and implemented an $8 \times$ 8 -bit shift register memory using the CONNECT cell library and the SRL $2.5 \mathrm{kA} / \mathrm{cm}^{2} \mathrm{Nb}$ process. The total number of Josephson junctions including the test system is 4184 , and the circuit area is $2.1 \mathrm{~mm} \times 3.2 \mathrm{~mm}$. We measured the error rates of every storage node by reading out the data $2^{16}$ times at the clock frequency of $16 \mathrm{GHz}$. The measured error rates were lower than $10^{-10}$ with DC bias margin better than $\pm 5 \%$.
\end{abstract}

Index Terms-Bit-error rate, Josephson logic, Josephson memories, RSFQ circuits, superconducting integrated circuits.

\section{INTRODUCTION}

$\mathbf{R}$ APID-SINGLE-FLUX-QUANTUM (RSFQ) logic circuits are a promising technology for realizing future ultra-fast digital systems with low power consumption [1]. Recently, SFQ circuits such as microprocessors [2], [3] and network switches [4] have been successfully demonstrated at operating frequencies of a few tens of GHz. However, a lack of short access-time, high-density memories is an impediment to realize high-performance digital systems using RSFQ logic circuits. As a candidate for such a memory, we have been investigating RSFQ shift register memories [5], [6], in which the storage nodes are formed by using an array of high-density RSFQ shift registers and the data are accessed bit-serially. The advantages of the shift register memory are high throughput and low power consumption compared with the latching type Josephson memories [7], [8]. In addition, the shift register memory has good compatibility with our RSFQ microprocessor based on bit-slice architecture.

Although the circuit scale of RSFQ LSIs is increasing and recently reached tens of thousands of junction levels, we have come up against several issues in terms of the circuit yield in this

Manuscript received August 29, 2006. This work was supported by the New Energy and Industrial Technology Development Organization (NEDO) through ISTEC as a Collaborative Research and Superconductors Network Device Project.

T. Hikida, K. Fujiwara, and N. Yoshikawa are with the Department of Electrical and Computer Engineering, Yokohama National University, Yokohama, 240-8501 Japan (e-mail: yoshi@yoshilab.dnj.ynu.ac.jp).

A. Fujimaki is with the Department of Quantum Engineering, Nagoya University, Nagoya, 464-8603 Japan (e-mail: fujimaki@ nuee.nagoya-u.ac.jp).

$\mathrm{H}$. Terai is with the National Institute of Information and Communication Technology, Kobe, 651-2492 Japan (e-mail: terai@ nict.go.jp).

S. Yorozu is with the International Superconductivity Technology Center Superconductivity Research Laboratory, Tsukuba, 305-8501 Japan (e-mail: yorozu@istec.or.jp).

Color versions of one or more of the figures in this paper are available online at http://ieeexplore.ieee.org.

Digital Object Identifier 10.1109/TASC.2007.898689

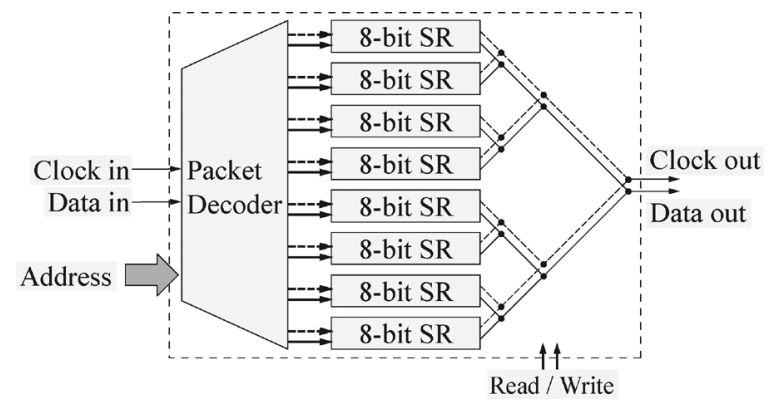

Fig. 1. Block diagram of an $8 \times 8$-bit shift register memory.

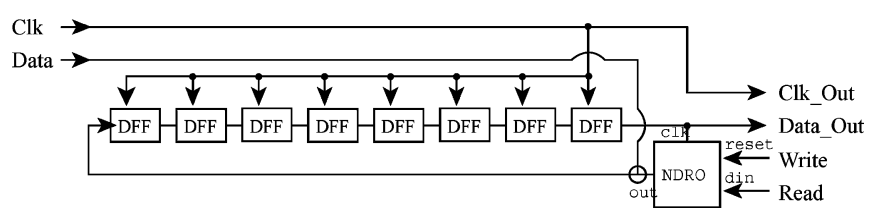

Fig. 2. Block diagram of the 8-bit shift register with a feedback loop for nondestructive read-out operation.

circuit scale. The circuit yield of large RSFQ circuits is thought to deteriorate for several reasons, such as defects, flux trapping, magnetic flux induced by large currents, timing jitter, and so on. Therefore, a systematic study on the reliability and stability of large RSFQ circuits is necessary to solve these issues. These circuits are good test circuits for investigating the reliability of large circuits because of their regularity and large circuit scale.

In this paper, we designed an error-rate measurement system and examined the error rates of an $8 \times 8$-bit shift register memory in order to demonstrate the reliability of the shift register memory.

\section{ERROR-RATE TEST SyStem FOR RSFQ SHIFT REGISTER MEMORIES}

Fig. 1 shows a block diagram of an $8 \times 8$-bit shift register memory. The memory is composed of a packet decoder, eight 8-bit shift registers (SR) with nondestructive read-out operation, and a tree of confluence buffers. The packet decoder switches the data and clock into the appropriate shift register specified by the 3-bit address. Fig. 2 shows a block diagram of the 8-bit shift register [6], where counter flow clocking is used to reduce the memory access time. The shift register has an internal feedback loop to achieve nondestructive read-out operation.

Fig. 3 shows a block diagram of the error-rate test system for the shift register memory. The test system is composed of a 16-bit pulse generator, a $16 \mathrm{GHz}$ clock generator $(\mathrm{CG})$ and a shift register memory. The error-rate measurement of the memory is carried out as follows. At first the memory is set to 


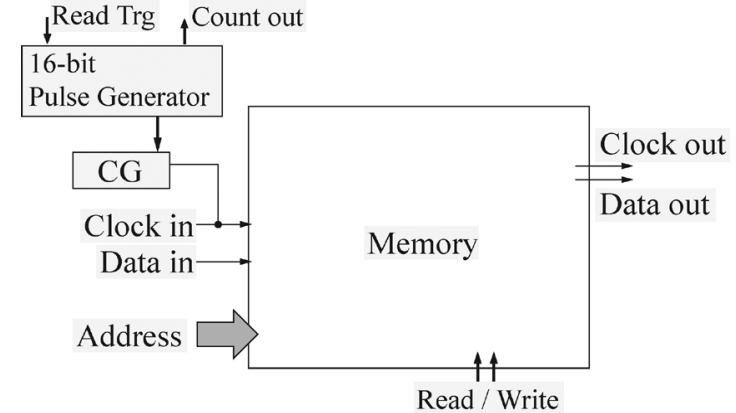

Fig. 3. Block diagram of the error-rate measurement system for the shift register memory.

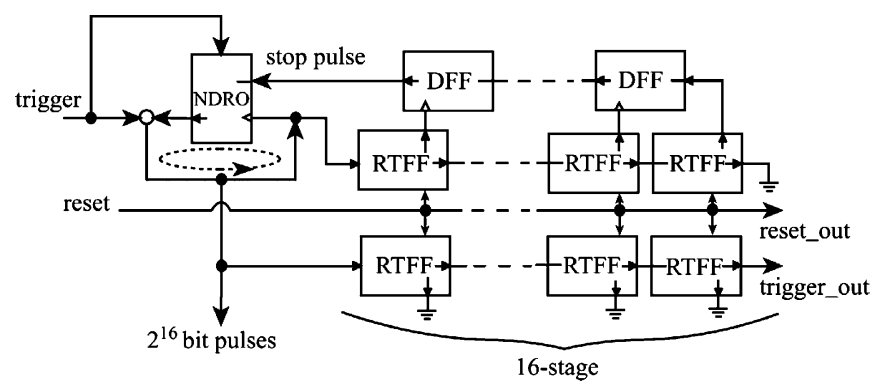

Fig. 4. Block diagram of the 16-bit pulse generator.

the write mode, and initial 8-bit data are loaded at low speed into the shift register specified by the memory address. Then the memory is switched to the read-out mode. When a trigger pulse (Read $\operatorname{Trg}$ ) is applied to the 16-bit pulse generator, it generates $2^{16}$ SFQ pulses and sends them to the CG. At each input of the SFQ pulse, the CG generates and sends eight clock pulses to the shift register memory at $16 \mathrm{GHz}$, and the 8-bit data stored in the shift register are read out $2^{16}$ times at high speed. Finally, the data in the memory are read out again at low speed and are compared with the initial data to check for errors. The frequency of the 16-bit pulse generator is $1 \mathrm{GHz}$, which is designed so that the cycle time is longer than the 8-bit read-out time of the shift register memory. The $2^{16}$-times read-out and the error check were repeated many times and the system error rate was evaluated.

Fig. 4 shows a block diagram of the 16-bit pulse generator, whose architecture is similar to that proposed previously [9]. When a trigger pulse is applied to the pulse generator, a re-settable nondestructive read-out (NDRO) cell is set to the "on" state, and an SFQ pulse starts to go around the Josephson transmission line (JTL) loop to generate high-speed SFQ pulses. The control circuit is composed of an array of re-settable $\mathrm{T}$ flip-flops (RTFF) and D flip-flops (DFF), which sends a stop pulse to the NDRO after counting up $2^{16}$ pulses. The CG for 8 clock pulses is a ladder-type high-speed clock generator [10] and is composed of an array of splitters and confluence buffers.

We designed and implemented an $8 \times 8$-bit shift register memory and the error-rate test system using the CONNECT cell library [11] and the SRL $2.5 \mathrm{kA} / \mathrm{cm}^{2} \mathrm{Nb}$ process [12]. Fig. 5 shows a photograph of the error-rate test system. Its layout size is $2.1 \mathrm{~mm} \times 3.2 \mathrm{~mm}$. The total number of Josephson junctions and the total bias current are 4184 and $515 \mathrm{~mA}$, respectively. Logic simulations indicate that its DC bias margin of the

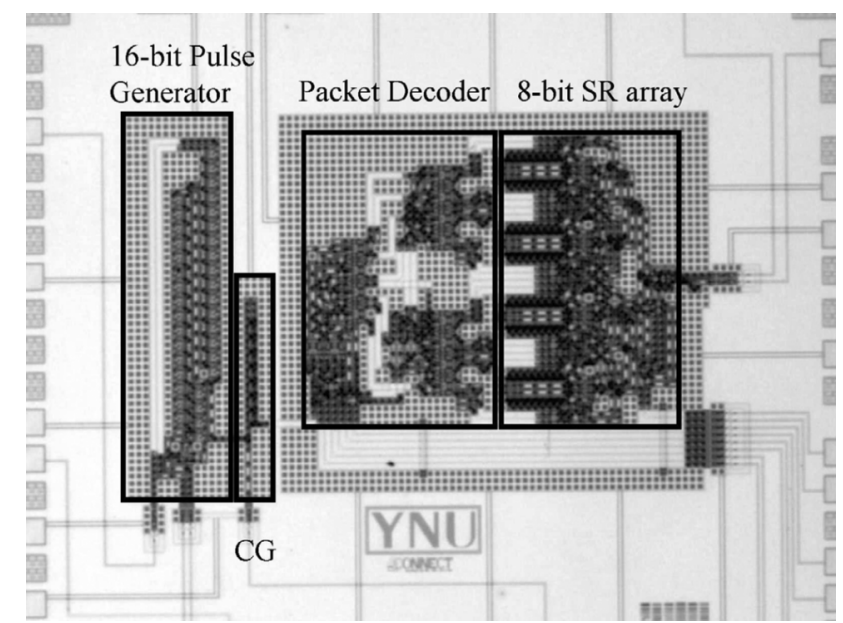

Fig. 5. Photograph of the error-rate measurement system for the $8 \times 8$-bit shift register memory implemented using the SRL $2.5 \mathrm{kA} / \mathrm{cm}^{2} \mathrm{Nb}$ standard process. The circuit size is $2.1 \mathrm{~mm} \times 3.2 \mathrm{~mm}$

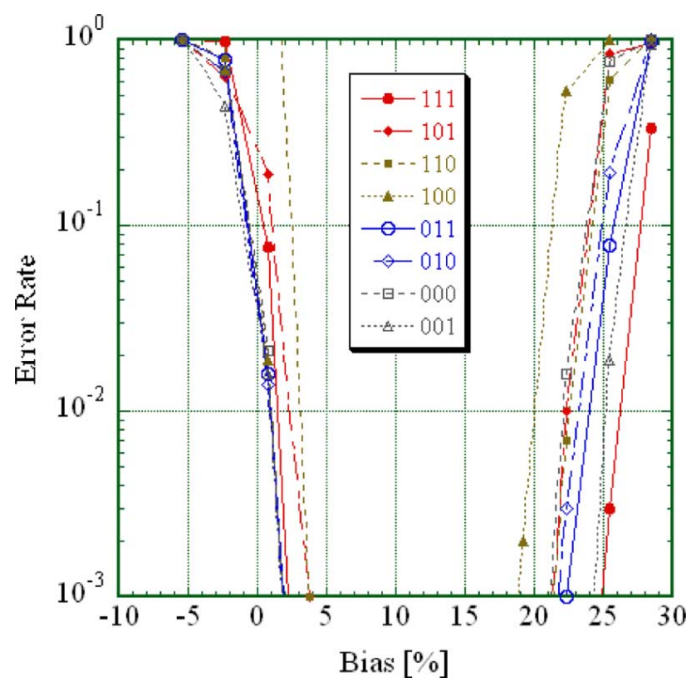

Fig. 6. The DC bias dependence of the system error rate of the $8 \times 8$-bit shift register memory for various memory addresses. The clock frequency is $16 \mathrm{GHz}$, and the stored data pattern is " $11010001 . "$

memory is $-20 \% \sim+25 \%$. The bias current feeding line of the CG is separated from the other circuit blocks, so that the frequency of the CG can be varied independently by changing its bias current.

\section{EXPERIMENTAL RESULTS}

Fig. 6 shows the DC bias dependence of the system error rate of the $8 \times 8$-bit shift register memory for various memory addresses, where the clock frequency is $16 \mathrm{GHz}$, and the stored data pattern is "11010001." It can be seen that the DC bias margin for the system error rate of $10^{-3}$ is better than $\pm 5 \%$ for every memory address.

Fig. 7 shows the DC bias dependence of the system error rate of the memory for various data patterns at $16 \mathrm{GHz}$ for the different samples. In Fig. (7a), the number "1" of the stored data is changed. In Fig. (7b), the number "1" of the data is the same but the data pattern is changed. One can see that the system error rate is affected by the stored data pattern, though the narrowest 


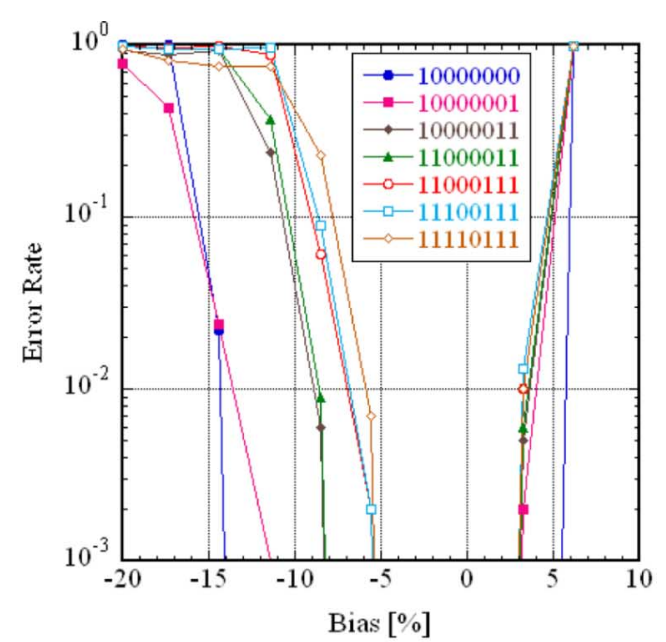

(a)

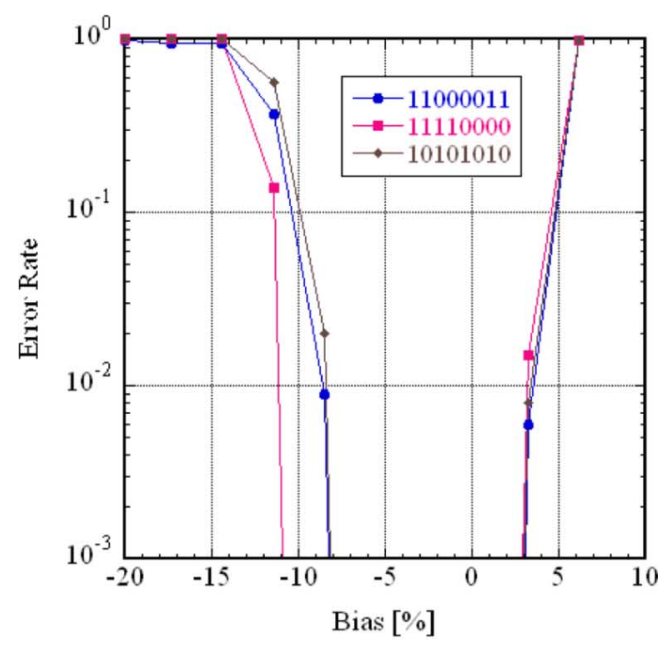

(b)

Fig. 7. The DC bias dependence of the system error rate of the $8 \times 8$-bit shift register memory for various data patterns at $16 \mathrm{GHz}$. The memory address is "011." (a) The number " 1 " in the stored data is changed. (b) The number " 1 " is the same but the data pattern is changed.

DC bias margin of $\sim \pm 4 \%$ is still sufficient for the circuit operation. This data pattern dependence is due to the data-dependent variation of the timing of the DFF and NDRO used in the shift register.

Fig. 8 shows the DC bias dependence of the system error rate of the memory for various clock frequencies, where the memory address is "011," and the stored data pattern is " 11010001 ." One can see that the system error rate gradually increases with an increase of clock frequency, especially at lower bias conditions. This upper bound of the operating frequency is limited by the timing in the feedback loop of the shift register.

Fig. 9 shows the DC bias dependence of the bit-error-rate (BER) of the memory cell of the shift register at $16 \mathrm{GHz}$ for the address " 011 " and the data pattern "11010001." The BER of the memory cell is calculated by dividing the system error rate by the data length of the shift register and the read-out times in a single error test, i.e., 8 and $2^{16}$. It can be seen from the figure that the BER of the memory cell is better than $10^{-10}$ with the DC bias margin $\pm 5 \%$.

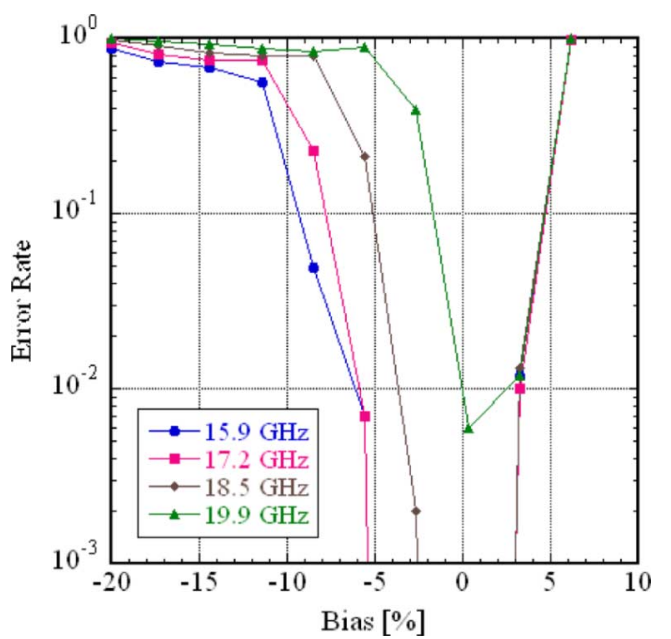

Fig. 8. The DC bias dependence of the system error rate of the $8 \times 8$-bit shift register memory for various clock frequencies. The memory address is "011," and the stored data pattern is " 11010001 ."

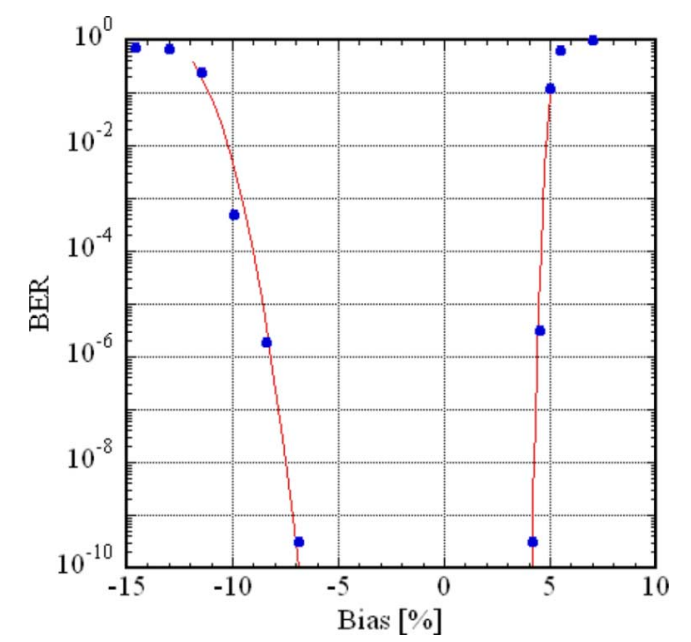

Fig. 9. The DC bias dependence of the bit-error-rate of the memory cell of the $8 \times 8$-bit shift register memory at $16 \mathrm{GHz}$ for the address " 011 " and the data pattern "11010001." The fitting curves are obtained by using Eq. (1).

\section{DISCUSSION}

The BER of a small circuit element is theoretically given by

$$
B E R=\frac{1}{2} \cdot \operatorname{erf} c\left[\sqrt{\frac{R}{8 k_{B} T B}}\left|I_{b}-I_{m}\right|\right],
$$

where $R$ is the resistance of the system under consideration, $k_{\mathrm{B}}$ is the Boltzman constant, $T$ is the effective temperature, $B$ is the bandwidth of the system, $I_{\mathrm{b}}$ is the bias current, and $I_{\mathrm{m}}$ is the bias current where the error rate becomes 0.5 [13], [14]. In Fig. 9, we plot the fitting curves obtained from (1). One can see that the agreement between the measured and calculated results is quite good. If we extrapolate the fitting curves, we can estimate that the DC bias margin is better than $\pm 2.5 \%$ for the BER of $10^{-30}$, which is required for digital applications.

\section{CONCLusions}

We designed a error-rate test system for RSFQ shift register memories to investigate the reliability of large RSFQ logic circuits. The dependence of the system error rate on the memory 
address, the data pattern, and the clock frequency was examined. The measured bit-error-rate of the memory cell of the $8 \times 8$-bit shift register memory is lower than $10^{-10}$ with DC bias margin better than $\pm 5 \%$.

\section{REFERENCES}

[1] K. K. Likharev and V. K. Semenov, "RSFQ logic/memory family: A new Josephson-junction technology for sub-terahertz-clock frequency digital systems," IEEE Trans. Appl. Supercond., vol. 1, pp. 1-28, Mar. 1991.

[2] M. Tanaka, F. Matsuzaki, T. Kondo, N. Nakajima, Y. Yamanashi, A. Fujimaki, H. Hayakawa, N. Yoshikawa, H. Terai, and S. Yorozu, "A single-flux-quantum logic prototype microprocessor," in Tech. Dig. IEEE Int. Solid-State Circuit Conf., San Francisco, CA, Feb. 2004.

[3] M. Tanaka, T. Kondo, N. Nakajima, T. Kawamoto, Y. Yamanashi, Y. Kamiya, A. Akimoto, A. Fujimaki, H. Hayakawa, N. Yoshikawa, H. Terai, Y. Hashimoto, and S. Yorozu, "Demonstration of a singleflux-quantum microprocessor using passive transmission lines," IEEE Trans. Appl. Supercond., vol. 15, pp. 400-404, 2005.

[4] S. Yorozu, Y. Kameda, Y. Hashimoto, H. Terai, A. Fujimaki, and N. Yoshikawa, "Progress of single flux quantum packet switch technology," IEEE Trans. Appl. Supercond., vol. 15, pp. 411-414, June 2005.

[5] K. Fujiwara, Y. Yamashiro, N. Yoshikawa, A. Fujimaki, H. Terai, and S. Yorozu, "Design and high-speed test of $(4 \times 8)$-bit single-flux-quantum shift register files," Supercond. Sci. Technol., vol. 16, pp. 1456-1459, 2003.
[6] K. Fujiwara, Y. Yamashiro, N. Yoshikawa, Y. Hashimoto, S. Yorozu, H. Terai, and A. Fujimaki, "High-speed test of SFQ-shift register files using PTL wiring," Physica C, vol. 412-414, pp. 1586-1590, 2004.

[7] S. Nagasawa, H. Hasegawa, T. Hashimoto, H. Suzuki, K. Miyahara, and Y. Enomoto, "Superconducting latching/SFQ hybrid RAM," IEEE Trans. Appl. Supercond., vol. 11, pp. 533-536, Mar. 2001.

[8] A. F. Kirichenko, S. Sarwana, D. K. Brock, and M. Radpavar, "Pipelined DC-powered SFQ RAM," IEEE Trans. Appl. Supercond., vol. 11, no. 1, pp. 537-540, Mar. 2001.

[9] A. F. Kirichenko, O. Mukhanov, and A. Ryzhikh, "Advanced on-chip test technology for RSFQ circuits," IEEE Trans. Appl. Supercond., vol. 7, pp. 3438-3441, Jun. 1997.

[10] Z. J. Deng, N. Yoshikawa, S. R. Whiteley, and T. Van Duzer, "Datadriven self-timed RSFQ digital integrated circuit and system," IEEE Trans. Appl. Supercond., vol. 7, pp. 3634-3637, June 1997.

[11] S. Yorozu, Y. Kameda, H. Terai, A. Fujimaki, T. Yamada, and S. Tahara, "A single flux quantum standard logic cell library," Physica C, vol. 378-381, pp. 1471-1474, 2002.

[12] S. Nagasawa, Y. Hashimoto, H. Numata, and S. Tahara, "A 380 ps, 9.5 $\mathrm{mW}$ Josephson 4-Kbit RAM operated at a high bit yield," IEEE Trans. Appl. Supercond., vol. 5, pp. 2447-2452, 1995.

[13] Q. P. Herr and M. J. Feldman, "Error rate of RSFQ circuit: Theory," IEEE Trans. Appl. Supercond., vol. 7, pp. 2661-2664, Jun. 1997.

[14] A. M. Herr, M. J. Feldman, and M. F. Bocko, "Timing jitter and bit errors in a 64-bit circular shift register," IEEE Trans. Appl. Supercond., vol. 9, pp. 3721-3724, Jun. 1999. 